\title{
BCG Infection after Bladder Cancer Treatment-3 Clinical Case Reports
}

\author{
Joana Alves1, Angélica Ramos ${ }^{2,3}$, Teresa Carvalho², Susana Silva1', João Tiago Guimarães, ${ }^{2,3,4}$, \\ António Sarmento ${ }^{1}$ \\ ${ }^{1}$ Infectious Diseases Department, Faculty of Medicine, University of Porto, Centro Hospitalar São João EPE, \\ Porto, Portugal \\ ${ }^{2}$ Clinical Pathology Department, Centro Hospitalar São João EPE, Porto, Portugal \\ ${ }^{3}$ EPI Unit, Institute of Public Health of University of Porto, Porto, Portugal \\ ${ }^{4}$ Biochemistry Department, Faculty of Medicine, University of Porto, Porto, Portugal \\ Email: joanamargaridaalves@gmail.com
}

Received 6 November 2015; accepted 22 December 2015; published 25 December 2015

Copyright (C) 2015 by authors and Scientific Research Publishing Inc.

This work is licensed under the Creative Commons Attribution International License (CC BY).

http://creativecommons.org/licenses/by/4.0/

c) (i) Open Access

\begin{abstract}
Immunotherapy with Bacillus Calmette-Guérin (BCG) to treat non-muscle invasive bladder cancer has become an effective and superior alternative to chemotherapy. Intravesical treatment with BCG appears to be relatively safe; however, occasionally BCG infection complicates such treatment. In the present work we describe three patients in whom BCG infection occurred after intravesical BCG therapy. All patients had positive urine culture for Mycobacterium tuberculosis complex, using AccuProbe culture identification and then Genotype Mycobacterium MTBC test identified Mycobacterium bovis BCG. The diagnosis is difficult and microbiologic study is usually negative, so high index of suspicion is essential.
\end{abstract}

\section{Keywords}

Bacillus Calmette-Guérin, Intravesical BCG Therapy, BCG Infection, Disseminated M. bovis BCG Infection

\section{Introduction}

Bacillus Calmette-Guérin (BCG), a live, attenuated strain of Mycobacterium bovis, interacts with the immune system to produce systemic immunity to BCG. $M$. bovis BCG has been traditionally used as a vaccine against tuberculosis. Further, intravesical administration of BCG has been shown to be effective and a superior alternative to chemotherapy in treating non-muscle invasive bladder cancer [1]. Intravesical treatment with BCG ap- 
pears to be relatively safe. In a series of 2026 treated patients, $<5 \%$ had major adverse reactions, which included granulomatous prostatitis, pneumonitis, hepatitis, sepsis, and hypersensitivity reactions [2]. Here, we report three cases of a disseminated M. bovis BCG infection that occurred after intravesical BCG therapy for bladder cancer.

Some components, such as multi-leveled equations, graphics, and tables are not prescribed, although the various table text styles are provided. The formatter will need to create these components, incorporating the applicable criteria that follow.

\section{Case Reports}

All patients had urothelial carcinoma of the bladder and were submitted to transurethral resection of bladder tumor (TURB). Two weeks after resection, they received intravesical instillations of the Tice strain of BCG.

All patients had lower urinary tract symptoms during and after treatment.

Patient 1, a 79-year-old man, had a history of anorexia, weight loss, night sweats and asthenia during BCG instillations. After 2 weeks of the last one, he began clinical features of fever, dyspnea and cough with mucous expectoration. Laboratory investigation with elevated inflammatory parameters; sterile piyuria in urine sample; $\mathrm{x}$-ray without infiltrations and some ground glass opacities on lung bases in torax CT scan. All initial etiological investigations were inconclusive including negative direct and cultural mycobacteriological study (urine, gastric juice and bronchial secretions). The patient fulfilled 7 days of ceftriaxone and 5 days of azithromycin for respiratory infection and had favorable outcome. After 3 months the patient returns, with persistence of asthenia, anorexia and weight loss. The study was repeated, bone marrow biopsy revealed granulomatous inflammatory process and 3 weeks later a strain of Mycobacterium tuberculosis complex (MTBc) in urine culture was isolated. After molecular differentiation within the complex, a strain of M. bovis BCG was identified (Figure 1).

The patient completed 12 months of isoniazid, rifampin and 2 months with ethambutol with favorable outcome.

Patient 2, an 85-year-old man, with history of terminal renal failure (polycystic kidney disease) in regular hemodialysis program had BCG instillations three years ago. At his follow-up visit on hemodialysis center, on physical examination was observed inflammatory signals in the central venous catheter (CVC) and elevated inflammatory parameters, therefore he started vancomycin and ceftazidime for probable catheter infection. Two days after, he was admitted for syncope. The patient was febrile; the inflammatory parameters were still elevated and had leucocyturia. Empirical antibioterapy with ciprofloxacin was started for urinary tract infection. Urine and blood cultures were negative. Eventually the patient started to improve but always remained with abundant sterile piyuria in all urine sediments. Further laboratory testing was performed-nucleic acid amplification was positive for MTBC and a valued strain of M. bovis BCG was isolated, both in urine. The patient was discharged with antituberculous therapy. After one month the patient had deteriorated clinically and was admitted to the hospital with sepsis due to an urinary infection. Blood and urine cultures were positive for $E$. coli. Despite therapy he eventually died.

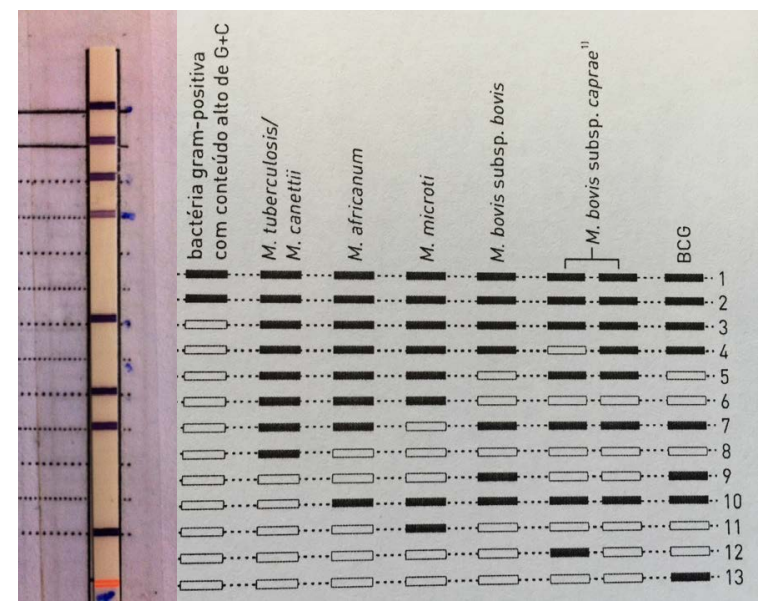

Figure 1. Patient $1 M$. bovis BCG identification by Genotype Mycobacterium MTBC $^{\circledR}$. 
After 9 months of BCG instillations, patient 3, a 78-year-old man, was again subjected to a transurethral resection for cancer recurrence and, after surgery the patient initiated clinical features of urinary tract infection. Piperacillin plus tazobactam was initiated and then replaced by imipenem for persistent fever. He developed sepsis with cardiovascular and renal dysfunction, and was transferred to the intensive care unit. In urine culture was isolated a strain of Enterococcus faecium multidrug-resistant and a strain of M. bovis BCG. He fulfilled 7 days of vancomycin showing clinical improvement. After resolution of this sepsis episode, he developed again hemodynamic deterioration with fever and elevated inflammatory parameters. A strain of S. epidermidis multidrug-resistant, sensitive to vancomycin, was isolated in blood culture. Started a new course of vancomycin for probable catheter infection. BCG infection was also assumed and antituberculosis treatment with isoniazid, rifampin and ethambutol was added. The patient improved and after 1 month was discharged.

Two months later, the patient died with sepsis due to an urinary tract infection. Klebsiella pneumonia was isolated in urine culture.

\section{Microbiology}

The samples (3 urines) were decontaminated according to the N-acetyl-L-cysteine-sodium hydroxide routine method [3]. The digested, decontaminated and concentrated samples were inoculated into Middlebrook 7H9 broth medium BBL ${ }^{\mathrm{TM}}$ MGITTM (Becton, Dickinson and Company (BD), New Jersey, U.S.), supplemented with BBL $^{\text {TM }}$ MGIT $^{\text {TM }}$ OADC (BD) enrichment and BBL ${ }^{\text {TM }}$ MGIT $^{\text {TM }}$ PANTA ${ }^{\text {TM }}$ antibiotic mixture (BD) for 42 days at $37^{\circ} \mathrm{C}$.

The positive urine cultures were examined by smear microscopy by Kinyoun's stain to examine acid-fast bacilli and/or presence of serpentine cords (Figure 2). In the presence of serpentine cords, to determine the species of the isolates the following routine molecular approaches were used: 1) AccuProbe Mycobacterium tuberculosis complex culture identification test (GenProbe, San Diego, CA), which relies on isothermal amplification of nucleic acids and on oligonucleotide probes complementary to 16S rRNA; 2) Genotype Mycobacterium MTBC test (HainLifescience GmbH, Nehren, Germany), a test based on the DNA-STRIP technology that permits the differentiation of MTBc members, was used according to the manufacturer's instructions (HainLifescience GmbH, Nehren, Germany).

\section{Discussion}

BCG is considered the treatment of choice for most patients with superficial bladder cancer following transurethral resection of bladder cancer [1].

In 1976 Morales et al. described the first use of BCG strain in intravesical instillations as an adjuvant for the treatment of superficial bladder cancer [4]. Since then, the first reports about complications appeared.

A retrospective study by Lamm et al. suggests that local adverse effects, including cystitis, fever; hematuria, and prostatitis are the most frequent complications, whereas extravesical complications are rare. Systemic side effects such as influenza-like symptoms, malaise and chills, sepsis, pneumonitis, hepatitis, cytopenia, rash, arthralgia and arthritis was also reported [5]. More recently, Pérez-Jacoiste Asín et al. analyzed 256 patients and eleven of them developed a systemic BCG infection; from revised literature, disseminated infection was the most common manifestation, reported in 97 cases, from a total of 282 patients [6].

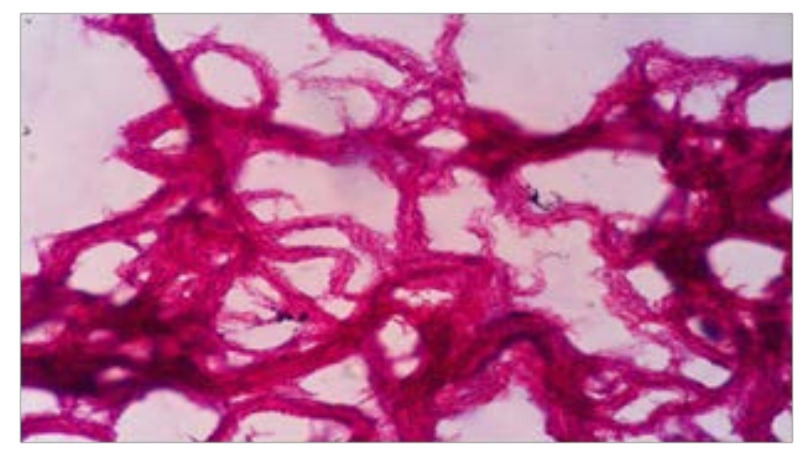

Figure 2. Cord formationin Kinyoun's stain. 
The diagnosis is difficult, urine and blood cultures are typically negative and treatment must be initiated based on high clinical suspicion. Tuncer et al. have detected M. bovis in the blood of patients with reactions following BCG treatment only in 9 of 126 samples [7]. Besides that, when urine cultures are positive is difficult to interpret whether is an inoculation or a treatment complication.

We have documented in present work three probable cases of BCG side effects after M. bovis BCG instillations with positive urine cultures. The diagnosis are challenging since symptoms are indistinct from other causes, so this must be part of the differential diagnosis, especially in patients with epidemiologic history.

Our 3 patients demonstrate the broad spectrum of syndromes of BCG infection-patiend 1 had lung disease that developed to disseminated BCG infection, patient 2 and 3 had cystitis. For patient 2 and 3 is difficult to know how much the BCG contributed for the sepsis.

When a complication of BCG treatment is suspected an endeavor to identify the strain should be made.

From the point of view of the laboratory we would like to call attention to these cases where we have a positive urine culture with AFB and/or serpentine cords in Kynioun stain that, subsequently, are identified as MTBc. Once these urine cultures may correspond to a patient under intravesical BCG therapy, we think that in such cases it is clinically important to discriminate within the complex.

The chance that BCG can be isolated through culture is affected by many factors, including the number of organisms present, the handling of samples and culture technique [8]. Moreover, highly sensitive and specific molecular techniques are needed to improve the chance that these mycobacteria will be isolated and distinguished within the MTBc. In the three cases here described $M$. bovis BCG was successfully isolated and identified from urine samples.

\section{Conclusions}

In summary, although rare, local and systemic complications after therapy with BCG for bladder cancer have been described, we must be aware of them when a patient with suggested clinical history is presented.

An attempt should be made to identify the causative agent and repeated if negative. Without an agent and since in BCG infection cultures are usually negative, it is important to remember that the diagnosis only requires a high index of suspicion.

Early complications diagnosis, preventing them when possible and managing them efficiently are essential.

\section{References}

[1] Babjuk, M., Böhle, A., Burger, M., Compérat, E., Kaasinen, E., Palou, J., Rouprêt, M., vanRhijn, B.W.G., Shariat, S., Sylvester, R. and Zigeuner, R. (2015) Guidelines on Non-Muscle-Invasive Bladder Cancer (Ta, T1 and CIS). European Association of Urology.

[2] Lamm, D.L. (1992) Complications of Bacillus Calmette-Guérin Immunotherapy. Urologic Clinics of North America, 19, 565-572.

[3] Kubica, G.P., Dye, W.E., Cohn, M.L. and Middlebrook, G. (1963) Sputum Digestion and Decontamination with NAcetyl-L-Cystein Sodium Hydroxide for Culture of Mycobacteria. American Review Respiratory Disease, 87, 775-779.

[4] Morales, A., Eidenger, D. and Bruce, A.W. (1992) Intracavitary Bacillus Calmette Guerin in the Treatment of Superficial Bladder Tumors. Journal of Urology, 116, 180-183.

[5] Lamm, D.L., van der Meijden, P.M., Morales, A., Brosman, S.A., Catalona, W.J., Herr, H.W., Soloway, M.S., Steg, A. and Debruyne, F.M. (1992) Incidence and Treatment of Complications of Bacillus Calmette-Guérin Intravesical Therapy in Superficial Bladder Cancer. Journal of Urology, 147, 596-600.

[6] Asín, M.A.P.-J., Fernández-Ruiz, M., López-Medrano, F., Lumbreras, C., Tejido, Á., Juan, R.S., Arrebola-Pajares, A., Lizasoain, M., Prieto, S. and Aguado, J.M. (2014) Bacillus Calmette-Guérin (BCG) Infection Following Intravesical BCG Administration as Adjunctive Therapy for Bladder Cancer. Medicine Jornal, 93, 236-254.

[7] Tuncer, S., Tekin, M.I., Ozen, H., Bilen, C., Unal, S. and Remzi, D. (1997) Detection of Bacillus Calmette-Guerin in the Blood by the Polymerase Chain Reaction Method of Treated Bladder Cancer Patients. Journal of Urology, 158, 2109-2112. http://dx.doi.org/10.1016/S0022-5347(01)68169-2

[8] Siatelis, A., Houhoula, D.P., Papaparaskevas, J., Delakas, D. and Tsakris, A. (2011) Detection of Bacillus CalmetteGuerin (Mycobacterium bovis BCG) DNA in Urine and Blood Specimens after Intravesical Immunotherapy for Bladder Carcinoma. Journal Clinical Microbiology, 49, 1206-1208. http://dx.doi.org/10.1128/JCM.01595-10 